\title{
The Streptomyces-Produced Antibiotic Fosfomycin Is a Promiscuous Substrate for Archaeal Isopentenyl Phosphate Kinase
}

\author{
Mark F. Mabanglo, ${ }^{\dagger}$ Adrian W. R. Serohijos, ${ }^{\dagger}$ and C. Dale Poulter ${ }^{*}{ }^{\dagger}$ \\ ${ }^{\dagger}$ Department of Chemistry, University of Utah, 315 South 1400 East, Salt Lake City, Utah 84112, United States \\ ${ }^{\ddagger}$ Department of Chemistry and Chemical Biology, Harvard University, 12 Oxford Street, Cambridge, Massachusetts 02138, United \\ States
}

Supporting Information

ABSTRACT: Isopentenyl phosphate kinase (IPK) catalyzes the phosphorylation of isopentenyl phosphate to form the isoprenoid precursor isopentenyl diphosphate in the archaeal mevalonate pathway. This enzyme is highly homologous to fosfomycin kinase (FomA), an antibiotic resistance enzyme found in a few strains of Streptomyces and Pseudomonas whose mode of action is inactivation by phosphorylation. Superposition of Thermoplasma acidophilum (THA) IPK and FomA structures aligns their respective substrates and catalytic

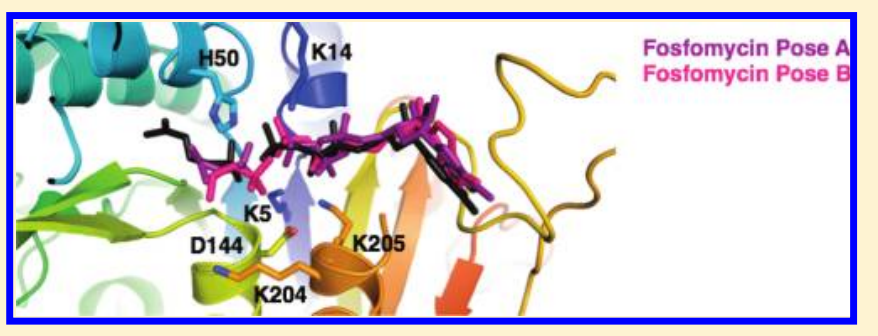
residues, including H50 and K14 in THA IPK and H58 and K18 in Streptomyces wedmorensis FomA. These residues are conserved only in the IPK and FomA members of the phosphate subdivision of the amino acid kinase family. We measured the fosfomycin kinase activity of THA IPK $\left[K_{\mathrm{m}}=15.1 \pm 1.0 \mathrm{mM}\right.$, and $\left.k_{\mathrm{cat}}=(4.0 \pm 0.1) \times 10^{-2} \mathrm{~s}^{-1}\right]$, resulting in a catalytic efficiency $\left(k_{\mathrm{cat}} / K_{\mathrm{m}}=2.6 \mathrm{M}^{-1} \mathrm{~s}^{-1}\right)$ that is 5 orders of magnitude lower than that of the native reaction. Fosfomycin is a competitive inhibitor of IPK $\left(K_{\mathrm{i}}=3.6 \pm 0.2 \mathrm{mM}\right)$. Molecular dynamics simulation of the IPK·fosfomycin $\cdot \mathrm{MgATP}$ complex identified two binding poses for fosfomycin in the IP binding site, one of which results in a complex analogous to the native IPK·IP.ATP complex that engages $\mathrm{H} 50$ and the lysine triangle formed by K5, K14, and K205. The other binding pose leads to a dead-end complex that engages K204 near the IP binding site to bind fosfomycin. Our findings suggest a mechanism for acquisition of FomA-based antibiotic resistance in fosfomycin-producing organisms.

$\mathrm{M}$ odern enzymes are commonly understood to be highly specific toward the substrates and chemical transformations that they catalyze. This selectivity underlies catalysis in biology. Enzyme selectivity prevents the conversion of unintended substrates that wastes cellular energy or has other deleterious effects on the organism in a cell where the chemical structures of metabolites are similar and where cross wiring of metabolic pathways exists. ${ }^{1}$ In contrast, primordial enzymes likely possessed a substantial degree of ambiguity with respect to binding and catalysis to generate a variety of products. ${ }^{2}$ These features are conducive to the evolution of new enzymes via gene duplication and optimization. ${ }^{2-4}$ In an organism under environmental stress, which potentially requires new reactions to survive, enhanced specificity can be selected by evolution. Low levels of promiscuous activities, i.e., those different than the main function, may persist in a specialized enzyme if they are not detrimental to the organism, and there is no selective pressure for their elimination. ${ }^{5}$ In some cases, promiscuous activity can complement gene deletions in metabolic pathways, for example, in Escherichia coli phn operon knockouts by recruitment of the phosphite-dependent hydrogenase activity of alkaline phosphatase, ${ }^{6}$ or E. coli auxotrophs that utilize promiscuous sugar kinases encoded by cryptic genes to rescue glucokinase deficient mutants. ${ }^{7}$ It has been suggested that promiscuity is an innate characteristic of all enzymes rather than an anomaly, and that there is a multitude of promiscuous activities extant in living cells available for exploitation by the host.

Recently, Mabanglo et al. reported the structures of isopentenyl phosphate kinase (IPK) in ternary complexes with its substrates and products. ${ }^{8}$ This enzyme is found only in Archaea, where it catalyzes the ATP-dependent phosphorylation of isopentenyl phosphate (IP) in the alternate mevalonate pathway (Scheme 1a). IPK is a member of the amino acid kinase (AAK) family of enzymes that phosphorylates small molecules with carboxylate, carbamate, phosphate, and phosphonate moieties. $^{8,9}$ In this family, IPK and the bacterial resistance protein fosfomycin kinase (FomA) in Streptomyces have a very high degree of structural homology despite significant sequence divergence $(22-25 \%$ identical) (Figure 1a). ${ }^{10}$ The two enzymes possess the lysine triangle and an active site histidine residue that are not found in other members of the phosphate/phosphonate subdivision (Figure 1b). ${ }^{8,9}$ Thus, it was suggested that the ipk gene was the source of fomA, presumably acquired by fosfomycin-producing strains of Streptomyces and Pseudomonas by horizontal gene transfer

Received: November 3, 2011

Revised: December 2, 2011

Published: December 9, 2011 
Scheme 1. Phosphoryl Transfer Reactions Catalyzed by IPK and FomA

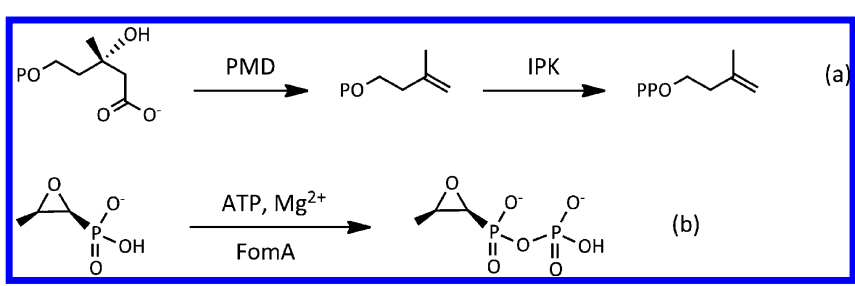

(a) The alternate route in the archaeal mevalonate pathway proceeds with the decarboxylation of mevalonate phosphate by phosphomevalonate decarboxylase (PMD) to form isopentenyl phosphate (IP), followed by phosphorylation by IPK to form the isoprene unit IPP. (b) FomA inactivates fosfomycin via phosphorylation using ATP as a donor. A second phosphorylation step by the enzyme FomB (not shown) completes the inactivating covalent modifications of the antibiotic.

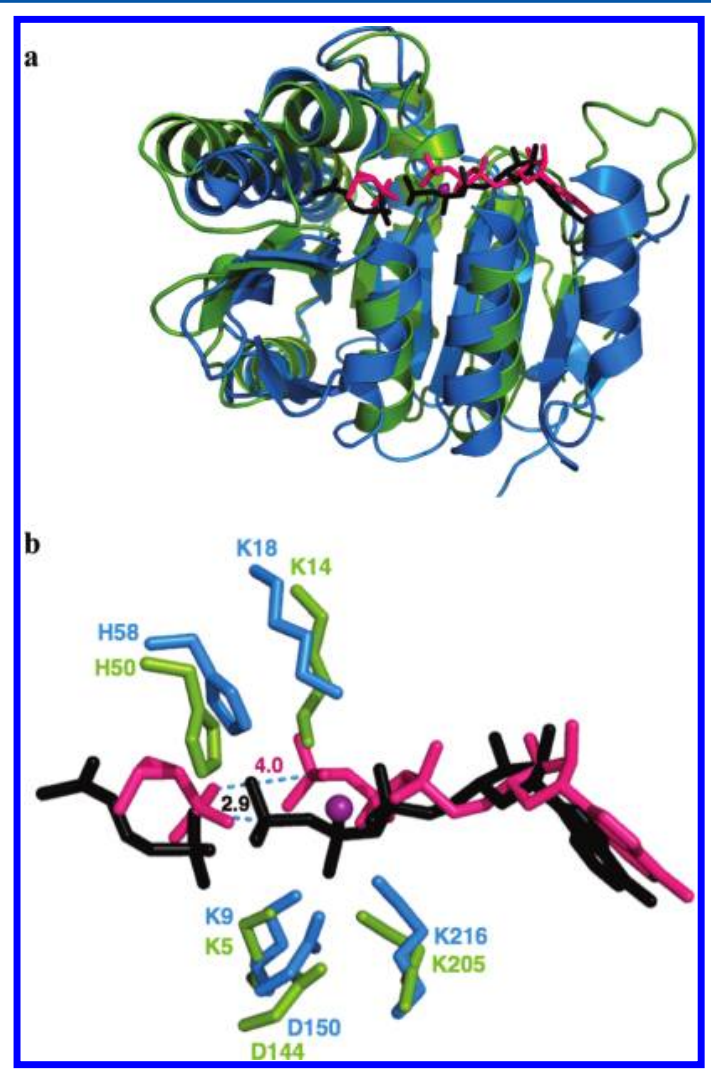

Figure 1. Thermoplasma acidophilum IPK and Streptomyces wedmorensis FomA have a high degree of structural homology. (a) Superimposed crystal structures of IPK (PDB entry 3LKK, green) and FomA (PDB entry 3QUO, blue) have a root-mean-square deviation of $2.6 \AA$ over 224 amino acid residues. The substrates of IPK (IP and ATP, black) and FomA (fosfomycin and ATP, pink) also align well in their respective active sites. The divalent metal $\mathrm{Mg}^{2+}$ is shown as a purple sphere. (b) Alignment of catalytic residues invariant in IPK and FomA, suggesting similar mechanisms of phosphoryl transfer. The FomA-fosfomycin-MgATP complex was modeled using the structures of the FomA.MgATP (PDB entry $3 \mathrm{QUN}$ ) and FomA.fosfomycin·ATP (PDB entry 3QUO) complexes. Distances between the nucleophilic oxygen atom of the phosphoryl group acceptor and electrophilic phosphorus atom of the terminal phosphate of ATP are indicated.

and converted into a specialized fosfomycin kinase through mutation and selection. ${ }^{8,11}$ This scenario is consistent with the acquisition of resistance in bacteria constantly exposed to an antibiotic. $^{12}$ In the case of Streptomyces, fosfomycin kinase activity was added to other resistance strategies in its arsenal (Scheme 1b), which includes fosfomycin inactivation by opening of the oxirane ring catalyzed by the metalloenzymes FosA, FosB, and Fos $\mathrm{X}^{13-16}$

We tested IPK from Thermoplasma acidophilum (THA IPK) for fosfomycin kinase activity and discovered that the antibiotic is an alternate substrate for the enzyme, albeit with poorer binding affinity and a reduced rate of turnover. Moreover, fosfomycin is a competitive inhibitor of isopentenyl phosphate kinase activity. Using molecular dynamics simulations, we discovered a binding mode for fosfomycin similar to that of IP that forms a stable complex with IPK, in addition to a separate binding mode that forms a dead-end complex.

\section{EXPERIMENTAL PROCEDURES}

Product Turnover Assay Using $\left[\gamma_{-}{ }^{32}\right.$ P]ATP. The phosphorylation of fosfomycin by IPK was visualized by incubating the enzyme in assay buffer [100 mM HEPES ( $\mathrm{pH}$ 7.5) containing $10 \mathrm{mM} \mathrm{MgCl}_{2}, 10 \mathrm{mM} \beta$-mercaptoethanol, 1 $\mathrm{mg} / \mathrm{mL}$ BSA, $\left[\gamma_{-}{ }^{32} \mathrm{P}\right] \mathrm{ATP}$, and varying concentrations of fosfomycin] at $37{ }^{\circ} \mathrm{C}$ for $10 \mathrm{~min}$. Each reaction was quenched with $113 \mu \mathrm{L}$ of a methanol/750 mM EDTA mixture (100:13, $\mathrm{v} / \mathrm{v})$. Samples $(5 \mu \mathrm{L})$ were spotted on silica plates and developed with a $\mathrm{CHCl}_{3} /$ pyridine/formic acid/ $\mathrm{H}_{2} \mathrm{O}$ mixture (30:70:16:10, v/v/v/v). The TLC plate was imaged for $24 \mathrm{~h}$ using a storage phosphor autoradiography cassette and visualized using a Typhoon 8600 variable mode imager (GE Healthcare). The same procedure was used in the parallel comparison of the products formed by Streptomyces wedmorensis FomA and THA IPK, except that $10 \mathrm{mM}$ fosfomycin and each enzyme at $10 \mu \mathrm{M}$ were used in the incubations.

MS/MS Fragmentation of the Fosfomycin Phosphate Product. The presence of fosfomycin phosphate was confirmed by performing MS/MS fragmentation on the ammonium form of fosfomycin phosphate (mass of 236) produced by THA IPK and S. wedmorensis FomA. The products were obtained by incubating each enzyme $(20 \mu \mathrm{M})$ in assay buffer [100 mM HEPES ( $\mathrm{pH} 7.5$ ), $1 \mathrm{mg} / \mathrm{mL}$ BSA, $10 \mathrm{mM} \beta$ mercaptoethanol, and $10 \mathrm{mM} \mathrm{MgCl}_{2}$ ] containing $25 \mathrm{mM}$ fosfomycin at $37{ }^{\circ} \mathrm{C}$ for $2 \mathrm{~h}$, in a total volume of $1 \mathrm{~mL}$. The enzymes were removed by centrifugation using a 10000 molecular weight cutoff Centricon, and the filtrate was flashfrozen in liquid $\mathrm{N}_{2}$ and lyophilized overnight in $1.5 \mathrm{~mL}$ microfuge tubes. The resulting slurry was dissolved in $100 \mu \mathrm{L}$ of $25 \mathrm{mM} \mathrm{NH}_{4} \mathrm{HCO}_{3}$ (pH 7.5) and subjected to MS/MS fragmentation.

Steady State Kinetics of the IPK-Fosfomycin Reaction. The protocol for fluorescent assays was based on the procedure of Pilloff et al. ${ }^{17}$ with modifications. The activities of coupling enzymes were determined by measuring the change in absorbance of $\mathrm{NADH}$ at $339 \mathrm{~nm}$. Different concentrations of lactate dehydrogenase (LDH) were mixed in assay buffer $(100$ $\mathrm{mM}$ HEPES containing $10 \mathrm{mM} \mathrm{MgCl}_{2}, 10 \mathrm{mM} \beta$ mercaptoethanol, $1 \mathrm{mg} / \mathrm{mL}$ BSA, $120 \mu \mathrm{M}$ pyruvate, and 150 $\mu \mathrm{M} \mathrm{NADH})$ at $37{ }^{\circ} \mathrm{C}$. For pyruvate kinase, different concentrations of the coupling enzyme were mixed in assay buffer (100 mM HEPES containing $10 \mathrm{mM} \mathrm{MgCl}_{2}, 10 \mathrm{mM} \beta$ mercaptoethanol, $1 \mathrm{mg} / \mathrm{mL}$ BSA, $1 \mathrm{mM}$ PEP, $4 \mathrm{mM}$ ADP, 150 $\mu \mathrm{M} \mathrm{NADH}$, and $\mathrm{LDH})$ at $37{ }^{\circ} \mathrm{C}$. The enzymatic rates in absorbance units per second were converted to specific activity units (units per milliliter) by using the NADH extinction coefficient $(\varepsilon)$ of $6.22 \mathrm{mM}^{-1} \mathrm{~cm}^{-1}$. To initiate the reaction, IPK 


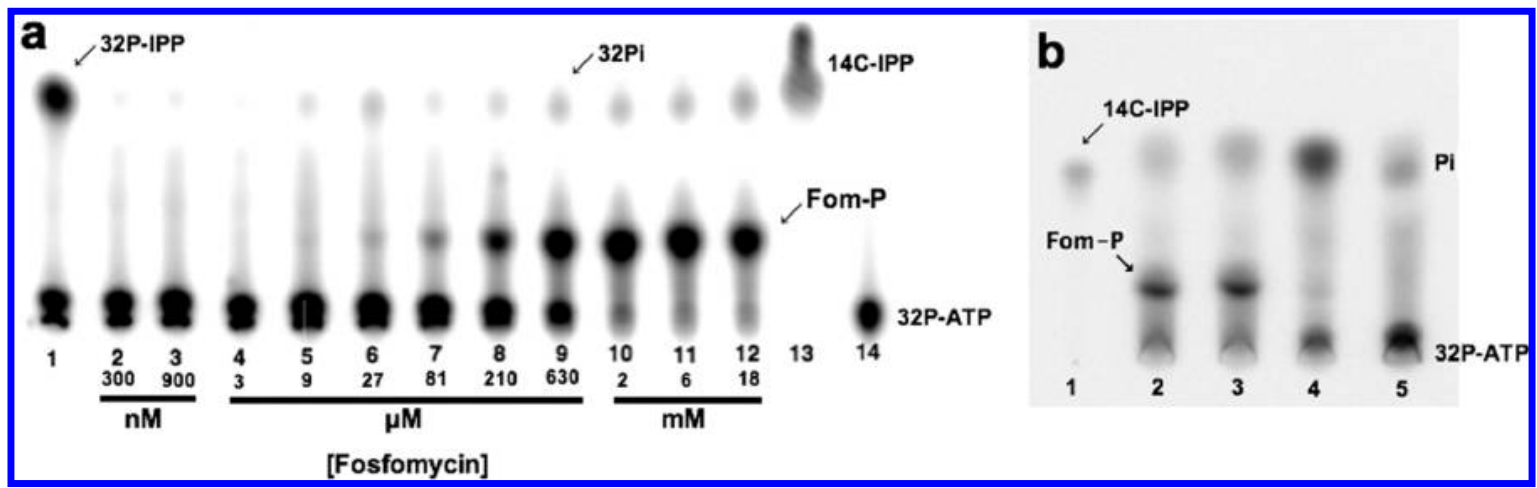

Figure 2. Autoradiograms showing that IPK can phosphorylate fosfomycin in the presence of ATP. (a) Product turnover assay of THA IPK using IP or fosfomycin as the substrate. The product of the native reaction, $\left[{ }^{32} \mathrm{P}\right] \mathrm{IPP}$, and the byproduct of the intrinsic ATPase activity, ${ }^{32} \mathrm{P}_{\mathrm{i}}$, migrate with almost similar $R_{f}$ values. This ATPase activity is independent of fosfomycin concentration. An increasing level of phosphorylation of fosfomycin is observed in the micromolar to millimolar range. All lanes except lane 13 contained $\left[\gamma^{-32} \mathrm{P}\right] \mathrm{ATP}$ and $200 \mathrm{nM}$ IPK: (1) $3.5 \mu \mathrm{M} \mathrm{IP}$ and IPK, (2) 300 nM fosfomycin, (3) $900 \mathrm{nM}$, (4) $3 \mu \mathrm{M}$, (5) $9 \mu \mathrm{M}$, (6) $27 \mu \mathrm{M}$, (7) $81 \mu \mathrm{M}$, (8) $210 \mu \mathrm{M}$, (9) $630 \mu \mathrm{M}$, (10) $2 \mathrm{mM},(11) 6 \mathrm{mM},(12) 18 \mathrm{mM}$, (13) $\left[{ }^{14} \mathrm{C}\right] \mathrm{IPP}$, and $(14)\left[\gamma_{-}{ }^{32} \mathrm{P}\right] \mathrm{ATP}$ and IPK. (b) Comparison of the products of $S$. wedmorensis FomA and THA IPK (20 $\mu \mathrm{M}$ each) upon incubation with fosfomycin. All lanes except lane 1 contained $\left[\gamma^{32} \mathrm{P}\right] \mathrm{ATP}:(1)\left[{ }^{14} \mathrm{C}\right] \mathrm{IPP}$ standard, (2) S. wedmorensis FomA and $10 \mathrm{mM}$ fosfomycin, (3) THA IPK and $10 \mathrm{mM}$ fosfomycin, (4) S. wedmorensis FomA incubated without fosfomycin, and (5) $10 \mathrm{mM}$ fosfomycin with neither $S$. wedmorensis FomA nor THA IPK.

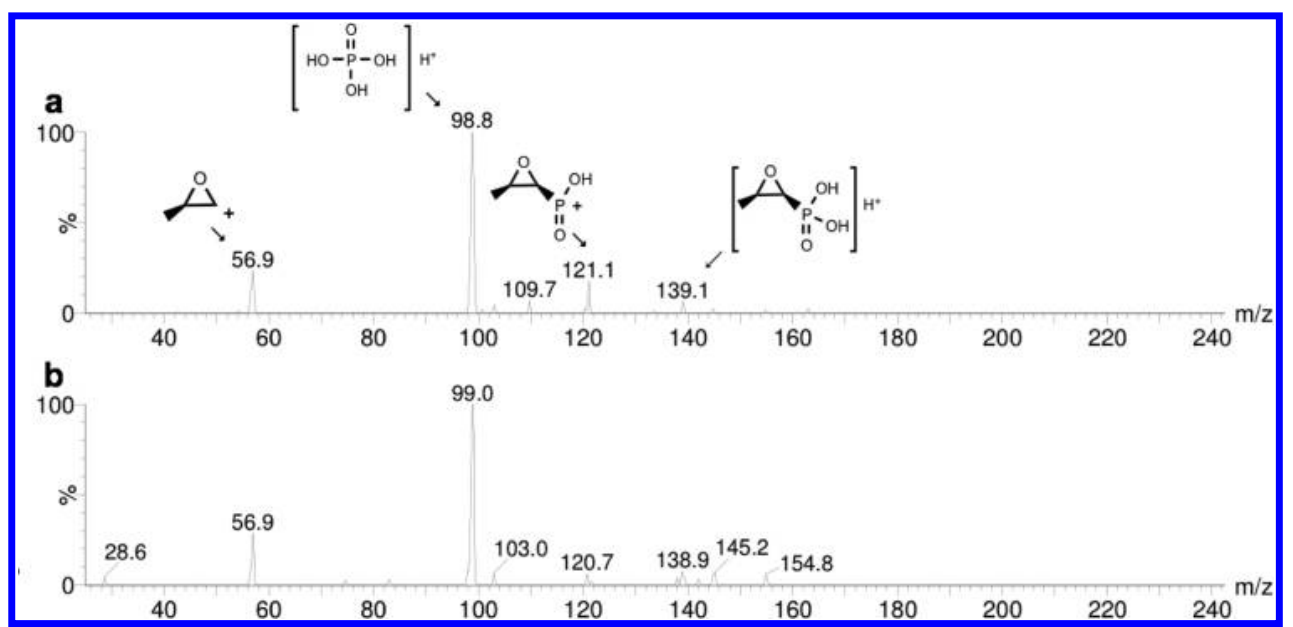

Figure 3. MS/MS fragmentation of the ammonium form of fosfomycin phosphate (mass of 236) produced by THA IPK and S. wedmorensis FomA. (a) Fragmentation of the product formed by THA IPK showed peaks corresponding to $\left[\mathrm{C}_{3} \mathrm{H}_{5} \mathrm{O}\right]^{+}$(mass of 56.9), $\left[\mathrm{H}_{4} \mathrm{O}_{4} \mathrm{P}\right]^{+}(\mathrm{mass}$ of 98.8 ), $\left[\mathrm{C}_{3} \mathrm{H}_{6} \mathrm{O}_{3} \mathrm{P}\right]^{+}$(mass of 121.1), and $\left[\mathrm{C}_{3} \mathrm{H}_{8} \mathrm{O}_{4} \mathrm{P}\right]^{+}$(mass of 139.1). The same peaks were found in the fragmentation product formed by $\mathrm{S}$. wedmorensis FomA (b).

was added to assay buffer [100 mM HEPES ( $\mathrm{pH} 7.5$ ) containing $10 \mathrm{mM} \mathrm{MgCl} 2,10 \mathrm{mM} \beta$-mercaptoethanol, and 1 $\mathrm{mg} / \mathrm{mL}$ BSA], including appropriate amounts of coupling enzymes and fosfomycin (or the native substrate IP for positive control reactions) in a final volume of $200 \mu \mathrm{L}$. The reaction was monitored at $37{ }^{\circ} \mathrm{C}$ for $600 \mathrm{~s}$ by observing the change in fluorescence $\left(\lambda_{\mathrm{ex}}=340 \mathrm{~nm} ; \lambda_{\mathrm{em}}=460 \mathrm{~nm}\right.$ ) (FluoroMax, Jobin Yvon Horiba). Background rates were measured at different concentrations of fosfomycin in the absence of the enzyme and were averaged. The initial rates were measured from the linear portion of the curve $(<15 \%$ consumption of the concentrationlimiting substrate). The kinetic constants were determined by fitting the matrices of initial rates to eq 1 using Grafit 5 (Erithacus Software): ${ }^{18}$

$$
\begin{aligned}
\nu= & V_{\max }[\mathrm{A}][\mathrm{B}] / K_{\mathrm{d}}{ }^{\mathrm{A}} K_{\mathrm{m}}{ }^{\mathrm{B}}+K_{\mathrm{m}}{ }^{\mathrm{B}}[\mathrm{A}]+K_{\mathrm{m}}{ }^{\mathrm{A}}[\mathrm{B}] \\
& +[\mathrm{A}][\mathrm{B}]
\end{aligned}
$$

where $\mathrm{A}$ and $\mathrm{B}$ are fosfomycin and ATP, respectively, $V_{\max }$ is the maximal rate, $K_{\mathrm{m}}$ is the Michaelis-Menten constant, and $K_{\mathrm{d}}$ is the enzyme dissociation constant. The lag time $\tau$ of the coupled assay is given by the equation

$$
\tau=K_{\mathrm{m}}^{\mathrm{ADP}} / V^{\mathrm{PK}}+K_{\mathrm{m}}^{\mathrm{Pyr}} / V^{\mathrm{LDH}}
$$

where $K_{\mathrm{m}}{ }^{\mathrm{ADP}}=0.3 \mathrm{mM}$ according to Sigma, $K_{\mathrm{m}}{ }^{\mathrm{Pyr}}=0.164 \mathrm{mM}$ according to Zewe and Fromm, ${ }^{19}$ and $V$ is the activity of the enzyme in units per milliliter. The lag time $\tau$ of the reaction was then set to $<10 \mathrm{~s}$, which ensured that the system reached $99 \%$ of the steady state rate of ADP production in $45 \mathrm{~s}$ according to the equation

$$
\mathrm{d} P / \mathrm{d} t=\nu_{0}\left(1-\mathrm{e}^{-t / \tau}\right)
$$

where $\nu_{0}$ is the steady state rate and $t$ and $\tau$ are in minutes.

Molecular Dynamics Simulations of the IPK.fosfomycin-MgATP Complex. We performed molecular dynamics simulations using GROMACS ${ }^{20}$ with CHARMM $27^{21}$ force field for proteins and small molecules ${ }^{22}$ and TIP $3 \mathrm{P}^{23}$ for water. The initial structure of the kinase is derived from the crystal structure of IPK, while the specific binding pose of ligands in 

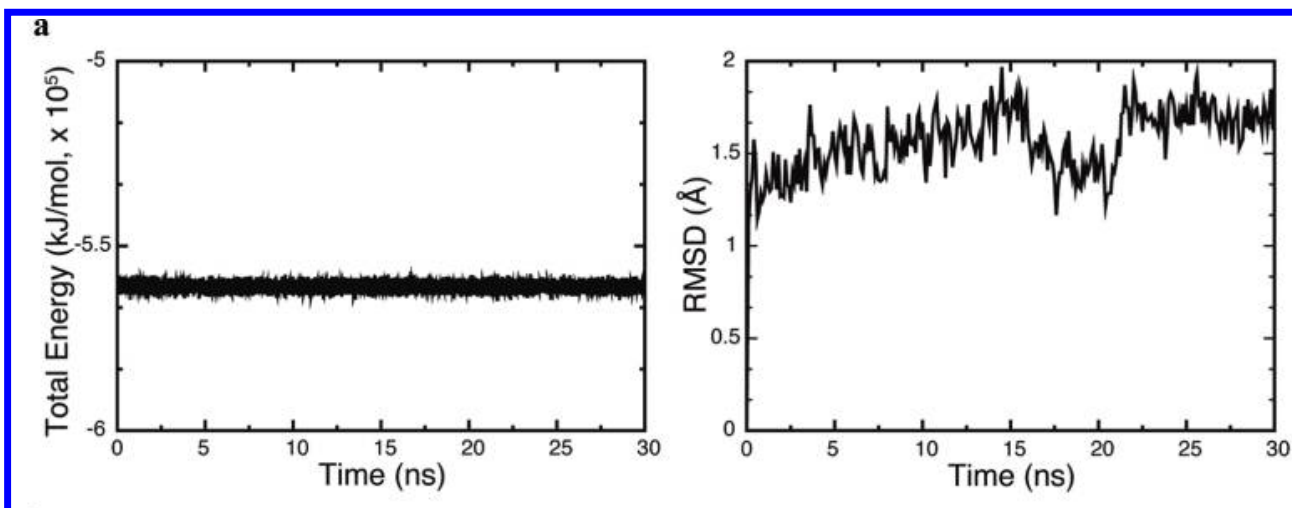

b
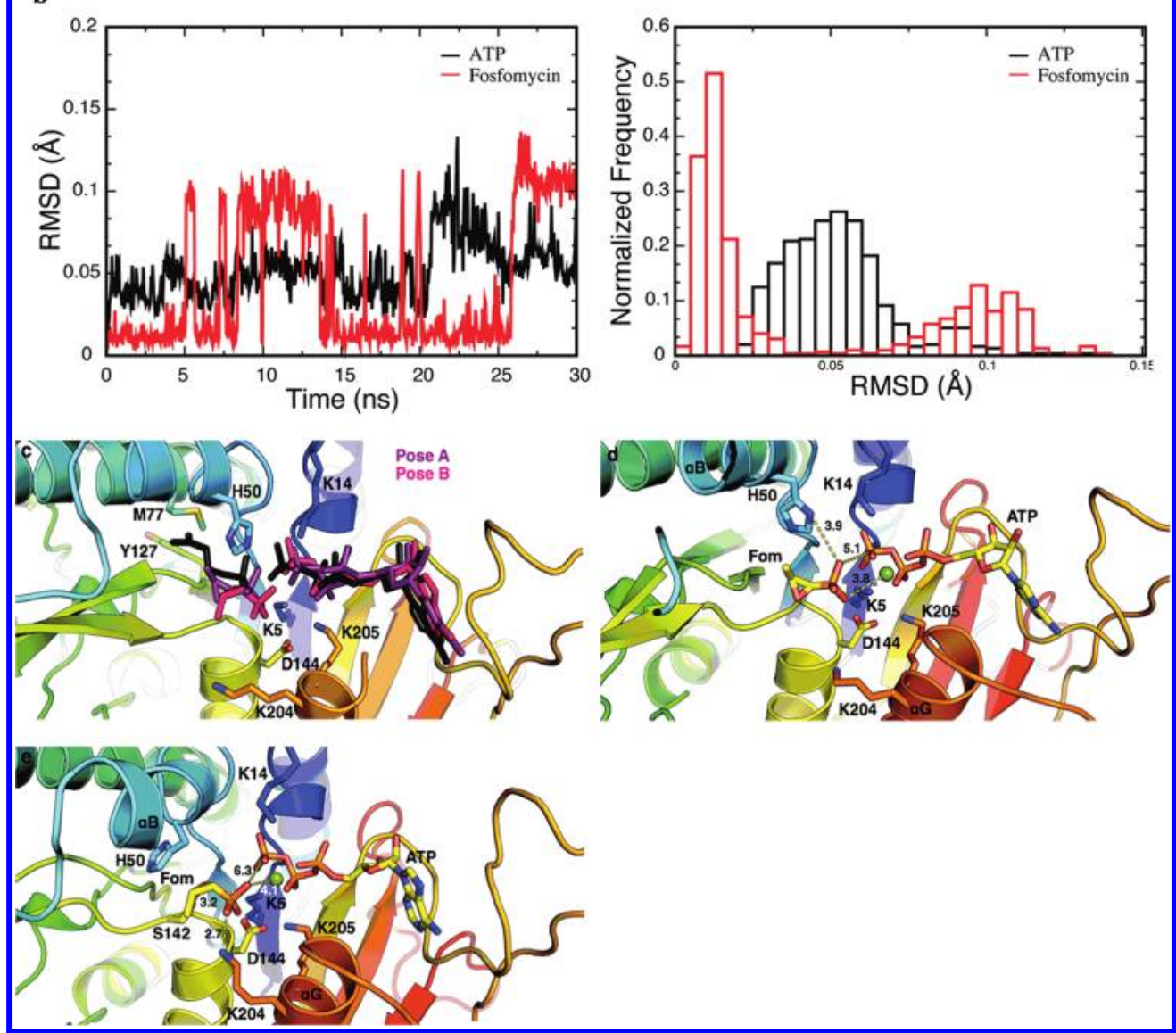

Figure 4. IPK·fosfomycin·MgATP complex that is stable with two binding poses for fosfomycin in the IP binding site. (a) Energy (left) and rmsd time series (right) showing that the modeled IPK·fosfomycin·MgATP complex is stable. (b) Ligand (fosfomycin and ATP, left) rmsd and histogram (right) showing a two-state binding conformation for fosfomycin, while ATP is essentially fixed. (c) Superposition of the native IPK.IP·ATP complex (substrates IP and ATP colored black) with the two binding poses of fosfomycin near the IP binding site. In both binding poses, fosfomycin is translated by an average of $4 \AA$ to relieve unfavorable interactions with the hydrophobic IP binding site. M77 and Y127 immediately below the aliphatic tail of IP are shown. (d) Productive IPK·fosfomycin·MgATP complex, similar to the native IPK.IP.ATP complex, with Mg ${ }^{2+}$ and the phosphonate group engaging in electrostatic interactions. (e) Dead-end complex in which S142 and K204 donate hydrogen bonds to fosfomycin, forming a stable complex.

the binding pocket of the IPK·fosfomycin·MgATP complex was derived from the superposition of PDB entries 3LKK (IPK) and 3D41 (FomA). The missing loop and $\alpha \mathrm{F}$ helix of IPK (residues 189-201) were modeled on the basis of sequence and structure alignment with NAGK (PDB entry 1GS5), ${ }^{24}$ where the corresponding secondary structures in the latter most approximate the length of these missing regions (Figures S1 and S2 of the Supporting Information). The geometry of the modeled secondary structures was validated using Molprobity. ${ }^{25}$ After an initial rapid energy minimization, the system was equilibrated by performing a position-restrained NVT simulation for 100 ps followed by an NPT run for an additional 100 ps. We then performed an unconstrained production run for $30 \mathrm{~ns}$ to validate the initially hypothesized poses of fosfomycin and MgATP. Long-range electrostatics was treated with particle mesh Ewald ${ }^{26}$ with a grid spacing of $12 \AA$ and a cutoff of $10 \AA$. We used the modified Berendsen ${ }^{27}$ and Parrinello-Rahman ${ }^{28}$ algorithms for temperature and pressure couplings, respectively. The same experiments were performed on the native IPK.IP·MgATP complex as a control. 


\section{RESULTS}

Detection of Fosfomycin Kinase Activity. Autoradiography studies using $\left[\gamma_{-}{ }^{32} \mathrm{P}\right] \mathrm{ATP}$ showed that fosfomycin is an alternate substrate for IPK. The formation of the product was accompanied by a concomitant decrease in the level of $\left[\gamma_{-}{ }^{32} \mathrm{P}\right]$ ATP when the concentration of fosfomycin was in the high micromolar to millimolar range (Figure 2a). In contrast, $K_{\mathrm{m}}^{\mathrm{IP}}=4.4 \mu \mathrm{M}$ for THA IPK. The product of the promiscuous reaction, assigned as fosfomycin phosphate, is consistent with the intense spot with an $R_{f}$ of 0.14 relative to IPP $\left(R_{f}=0.37\right)$. This spot did not appear in control reaction mixtures containing only IP or $\left[\gamma_{-}^{32} \mathrm{P}\right] \mathrm{ATP}$ and IPK. In addition, parallel incubation of $S$. wedmorensis FomA and IPK showed product spots with the same $R_{f}$ values given above (Figure $2 \mathrm{~b}$ ). These product spots were not found in incubations containing neither enzymes nor the fosfomycin substrate. MS/MS fragmentation of the ammonium form of fosfomycin phosphate (mass of 236) from incubations with THA IPK (Figure 3a) and S. wedmorensis FomA (Figure $3 \mathrm{~b}$ ) resulted in identical fragmentation patterns. Positive ion species corresponding to $\left[\mathrm{C}_{3} \mathrm{H}_{5} \mathrm{O}\right]^{+}$(mass of 57), $\left[\mathrm{C}_{3} \mathrm{H}_{6} \mathrm{O}_{3} \mathrm{P}\right]^{+}$(mass of 121 ), $\left[\mathrm{H}_{4} \mathrm{O}_{4} \mathrm{P}\right]^{+}$(mass of 99), and $\left[\mathrm{C}_{3} \mathrm{H}_{8} \mathrm{O}_{4} \mathrm{P}\right]^{+}$(mass of 139) were found in the fragmentations of the products of both enzymes. The two cations, $\left[\mathrm{C}_{3} \mathrm{H}_{5} \mathrm{O}\right]^{+}$and $\left[\mathrm{C}_{3} \mathrm{H}_{6} \mathrm{O}_{3} \mathrm{P}\right]^{+}$, were also observed in the fragmentation of a fosfomycin standard (Figure S3 of the Supporting Information).

In ${ }^{31} \mathrm{P}$ NMR assays, resonances corresponding to a phosphonate-phosphate moiety were not detected because of the slow reaction and an apparently unfavorable equilibrium between the fosfomycin.ATP and fosfomycin phosphate-ADP species. We initially attempted to use longer incubation times without success. The autoradiogram shows a byproduct of the kinase reaction whose $R_{f}$ is similar to that of IPP (Figure 2a), which is more pronounced in the absence of fosfomycin (Figure $2 b$, lane 4 ). Chen and Poulter ${ }^{11}$ had previously shown that this byproduct is ${ }^{32} \mathrm{P}_{\mathrm{j}}$, resulting from the combined effects of $\left[\gamma_{-}{ }^{32} \mathrm{P}\right]$ ATP degradation and the intrinsic ATPase activity of IPK. ATPase activity has been observed in many kinases and is commonly negligible compared to native kinase activities, ${ }^{29}$ although in some cases it is sufficiently large to interfere with their precise measurements. ${ }^{30}$ The ATPase activity of THA IPK was found to be sufficiently small ( 200 times slower) that it did not interfere with measurements of the promiscuous fosfomycin kinase activity.

Kinetic Constants. Initially, a series of fosfomycin concentrations from $750 \mu \mathrm{M}$ to $80 \mathrm{mM}$ was used to measure the apparent kinetic constants of the reaction at a saturating ATP concentration of $250 \mu \mathrm{M}$ (data not shown) to identify a suitable range of concentrations for kinetic measurements. Initial rates were fitted to the equation for bisubstrate reactions (Figure S4a of the Supporting Information) and were transformed to a Lineweaver-Burk plot (Figure S4b of the Supporting Information). The fosfomycin kinase reaction proceeds by the same sequential mechanism seen for IP and ATP. The kinetic constants for phosphorylation of IP and fosfomycin are summarized in Table 1 .

Fosfomycin Is a Competitive Inhibitor of IPK. Superposition of crystal structures of the THA IPK and FomA ternary complexes shows a close alignment of their respective IP and fosfomycin substrates (Figure $1 \mathrm{~b}$ ), suggesting that fosfomycin will be a competitive inhibitor of the faster IP kinase reaction. The best fit of our kinetic data was obtained with the equation for competitive inhibition (eq 1 of the Supporting Information). The Michaelis-Menten curves (Figure S5a of the Supporting Information) show an apparent increase in $K_{\mathrm{m}}{ }^{\mathrm{IP}}$ with an increase in inhibitor concentration, which was overcome at high concentrations of IP. The LineweaverBurk transformation of these curves showed typical competitive behavior (Figure S5b of the Supporting Information), with a $k_{\text {cat }}$ of $7.5 \mathrm{~s}^{-1}$ and a $K_{\mathrm{i}}$ of $3.6 \pm 0.20 \mathrm{mM}$. Weak inhibition of THA IPK by fosfomycin is consistent with the $K_{\mathrm{m}}$ for fosfomycin and is probably due to the incompatibility of its polar epoxide group with the hydrophobic pocket tailored for the isoprene unit in IP.

Binding Poses for FomA in IPK. Similar structures for the ternary complexes of IPK and FomA suggest that the native substrate IP and the promiscuous substrate fosfomycin adopt similar binding poses in IPK. To examine this issue, we performed molecular dynamics simulations on the IPK.fosfomycin-MgATP and IPK.IP.MgATP ternary complexes. To fix the MgATP ligand in its binding site during simulation, an accurately modeled structure for the missing $\alpha \mathrm{F}-\alpha \mathrm{G}$ loop and $\alpha$ F helix of IPK was supplied to the starting structure based on the aligned sequences of IPK from T. acidophilum and Methanothermobacter thermautotrophicus (MTH), ${ }^{8}$ S. wedmorensis FomA, ${ }^{10}$ and E. coli $\mathrm{NAGK}^{24}$ (Figures S1 and S2 of the Supporting Information). These alignments indicate that the secondary structure in NAGK, which differs from the sequence in THA IPK by only a single amino acid, can be used to model the missing loop and $\alpha \mathrm{F}$ helix in IPK. The system is stable and has an average $\mathrm{C} \alpha$ root-mean-square deviation (rmsd) of $1.5 \AA$ with respect to the starting structure (Figure 4a, left and right panels). We computed the heavy atom rmsd of fosfomycin and ATP and found two stable conformers of fosfomycin in the binding pocket (Figure $4 \mathrm{~b}$, left panel). The normalized rmsd distributions support the observed two-state conformations (Figure 4b, right panel). Closer inspection of these two conformations shows that fosfomycin binds to the $\mathrm{N}$-terminal lobe of THA IPK that contains the IP binding site (Figure 4c).

In both IPK.fosfomycin·MgATP structures, fosfomycin obstructs the IP binding site, as expected for a competitive inhibitor. Moreover, the MgATP conformation is essentially fixed, while fosfomycin is translated by $3.9 \AA$ in the first pose and $4.8 \AA$ in the second relative to the position occupied by IP, presumably to weaken unfavorable interactions between the polar epoxide and the hydrophobic binding pocket. In the first binding pose (Figure $4 \mathrm{~d}$ ), the oxygen of the epoxide is oriented toward the solvent region while the methyl group packs into the hydrophobic pocket. The catalytic residue H50 in the $\alpha \mathrm{B}$ helix forms a hydrogen bond with the phosphonate group, analogous to its interaction with IP. This places a phosphonate oxygen in fosfomycin $\sim 5.1 \AA$ from the $\gamma$-phosphorus in ATP. This distance is substantially longer than the $2.9 \AA$ distance between a nonbridging oxygen in IP and the $\gamma$-phosphorus of ATP in the IPK ternary complex (PDB entry 3LKK) and likely contributes to the low rate of the promiscuous reaction. However, the distance between the phosphonate oxygen and the $\gamma$-phosphorus of ATP in the structure of the FomA-fosfomycin.MgATP complex, modeled from coordinates of the FomA.MgATP (PDB entry 3QUN) and FomA.fosfomyci$\mathrm{n} \cdot \mathrm{ATP}$ (PDB entry $3 \mathrm{QUO}$ ) complexes, is $4.0 \AA$ (Figure $1 \mathrm{~b}$ ). ${ }^{31}$ Thus, it is likely that this distance is a good approximation of the real phosphonate $-\gamma$-phosphate distance. Examination of this binding pose also indicates that the active site $\mathrm{Mg}^{2+}$ is $3.7 \AA$ from a phosphonate oxygen, perhaps facilitating nucleophilic 
Table 1. Steady State Kinetic Constants for Phosphorylation of IP and Fosfomycin

\begin{tabular}{lccll} 
& IP·wild-type IPK & fosfomycin·wild-type IPK & IP·K204A & fosfomycin·K204A \\
$k_{\mathrm{cat}}\left(\mathrm{s}^{-1}\right)$ & $8.0 \pm 0.2$ & $(4 \pm 0.1) \times 10^{-2}$ & $7.7 \pm 0.2$ & $(3 \pm 0.1) \times 10^{-2}$ \\
$K_{\mathrm{m}}(\mu \mathrm{M})$ & $4.4 \pm 0.5$ & $(1.5 \pm 0.1) \times 10^{4}$ & $2.8 \pm 0.3$ & $(1.1 \pm 0.1) \times 10^{4}$ \\
$k_{\mathrm{cat}} / K_{\mathrm{m}}\left(\mathrm{M}^{-1} \mathrm{~s}^{-1}\right)$ & $1.8 \times 10^{6}$ & 2.6 & $2.8 \times 10^{6}$ & 2.5 \\
$K_{\mathrm{m}}{ }^{\mathrm{TTP}}(\mu \mathrm{M})$ & $6.0 \pm 0.5$ & $1.4 \pm 0.1$ & $6.9 \pm 0.6$ & $1.2 \pm 0.2$ \\
$K_{\mathrm{d}}(\mu \mathrm{M})$ & $4.6 \pm 1.5$ & $(1.8 \pm 0.3) \times 10^{4}$ & $4.6 \pm 1.3$ & $(1.5 \pm 0.5) \times 10^{4}$ \\
\hline
\end{tabular}

attack on ATP. This interaction as well as innate enzyme plasticity could help overcome the longer distance between reactive phosphonate and phosphate centers in the IPK.fosfomycin·MgATP complex. Interactions involving K5, K14, K205, and D144 observed in the IPK.IP.ATP crystal structure ${ }^{8}$ and those of corresponding residues in FomA ${ }^{31}$ are essentially conserved in this binding pose. Mutagenesis studies of these residues in FomA are consistent with their expected roles in binding and catalysis, ${ }^{31}$ suggesting that the first binding mode is a productive one.

In the second, less populated binding state, fosfomycin is moved even farther from the location of IP in the active site. The hydrogen bonding of the phosphonate moiety and H50 is absent. New hydrogen bonds are seen between the epoxide group and S142 and between a phosphonate oxygen and K204 in the $\alpha \mathrm{G}$ helix. In THA IPK, S142 binds a phosphate oxygen in IP. This residue is conserved in FomA and orients the phosphonate moiety in fosfomycin for nucleophilic attack on ATP. ${ }^{31}$ In addition, the closest phosphonate oxygen is $6.3 \AA$ from the $\gamma$-phosphorus in ATP and $4.1 \AA$ from $\mathrm{Mg}^{2+}$ in this second pose (Figure 4e).

Molecular dynamics simulation of the IPK.IP.MgATP complex showed that the complex is stable (Figure S6a of the Supporting Information) and revealed a single binding pose with IP and MgATP virtually unchanged from their respective poses in the crystal structure (Figure S6b of the Supporting Information). The latter result suggests that the crystal structure closely approximates the structure of the complex in solution and also lends credence to the two calculated binding poses of fosfomycin in the IPK active site.

The K204A Mutant IPK Has Fosfomycin Activity Comparable to That of Wild-Type IPK. Structures from crystallography and dynamics calculations suggest that K204 does not play a major role in catalysis. Recruitment of K204 to form a hydrogen bond to an oxygen atom of the phosphonate moiety in the second fosfomycin pose appears to replace the hydrogen bond between $\mathrm{H} 50$ and a phosphonate oxygen, leading to a dead-end complex . The $\mathrm{His}_{6}$-tagged K204A mutant was prepared, purified, and assayed for fosfomycin kinase activity. The mutant phosphorylated fosfomycin and the kinetic constants for the reaction were very similar to those for wild-type IPK (Table 1 and Figure S7a,b of the Supporting Information). In addition, mutation did not significantly alter the kinetic constants for the native reaction (Table 1$).^{11}$

\section{DISCUSSION}

Fosfomycin is a secondary metabolite produced in several species of Streptomyces and Pseudomonas ${ }^{32}$ that is used clinically to treat cystitis ${ }^{33}$ as well as infections by methicillin- and vancomycin-resistant strains of Staphylococcus aureus. ${ }^{34,35}$ The bactericidal activity of fosfomycin is based on the inhibition of MurA $^{36}$ by the irreversible alkylation of an active site cysteine residue, preventing the first step in peptidoglycan synthesis in which phosphoenolpyruvate is linked to the 3 '-hydroxyl group of UDP-N-acetylglucosamine. ${ }^{37}$ The resistance of bacteria to fosfomycin is achieved by mutations in MurA and by alterations in the glycerophosphate transporter. ${ }^{38,39}$ Fosfomycin is inactivated by hydrolysis of its epoxide moiety by the thiol transferases FosA and FosB, and the homologous hydratase enzyme FosX. ${ }^{13-16,40}$ Two additional enzymes, FomA and FomB, alter the antibiotic by two consecutive phosphorylation steps, resulting in a product that is unable to alkylate the relevant cysteine residue in MurA. The fom $A$ and fomB genes are found in the fosfomycin biosynthetic gene clusters of Streptomyces and Pseudomonas and are thought to confer resistance and facilitate transport in these hosts. ${ }^{41,42}$

The phosphate subdivision of the AAK family of enzymes consists of IPK, FomA, and uridine monophosphate kinase (UMPK). ${ }^{8,9,44}$ These three enzymes catalyze the transfer of a phosphoryl group from ATP by nucleophilic attack of an oxygen atom in a phosphate or phosphonate group in a reaction that proceeds through a pentavalent transition state. ${ }^{8,31,43}$ Within this subdivision, the crystal structures of the THA IPK-IP.ATP complex and the modeled structure of $S$. wedmorensis FomA-fosfomycin $\cdot M g A T P$ complexes showed excellent alignment of their catalytic residues and respective substrates. ${ }^{31}$ These similarities between IPK and FomA suggest that IPK might possess promiscuous activity toward fosfomycin, which could serve as the starting point for the evolution of fosfomycin resistance in Streptomyces and Pseudomonas following horizontal transfer of archaeal DNA.

THA IPK phosphorylates monophosphate esters with small isoprenoid and alkyl moieties. ${ }^{9,11}$ We found that the enzyme also phosphorylates the phosphonate moiety in fosfomycin. The catalytic constants for the reaction $\left(k_{\mathrm{cat}}=4 \times 10^{-2} \mathrm{~s}^{-1}\right.$, and $\left.K_{\mathrm{m}}=15 \mathrm{mM}\right)$ are substantially less favorable $\left(k_{\mathrm{cat}} / K_{\mathrm{m}}=2.6 \mathrm{M}^{-1}\right.$ $\left.\mathrm{s}^{-1}\right)$ than for phosphorylation of IP $\left(k_{\mathrm{cat}} / K_{\mathrm{m}}=1.8 \times 10^{6}\right)$. Although low, the catalytic efficiency for phosphorylation of fosfomycin by THA IPK could serve as starting point for the evolution of an enzyme with more potent fosfomycin kinase activity. There are several examples in the literature of enhancement of extrinsic activities by mutation of one or more residues in the active site. For instance, the E. coli RTEM $\beta$-lactamase increased its cephalosporinase activity 1.4 -fold and decreased its primary penicillase activity 3 -fold upon placement of the bacterial strain under the selective pressure of cephalosporin $\mathrm{C}$ through two mutations in the active site. ${ }^{44}$ Subsequently, other mutations were found to result in more dramatic enhancements in cephalosphorinase C activity. ${ }^{45-47}$

The low catalytic efficiency of phosphorylation of fosfomycin by IPK results primarily from the conformation of the molecule in the IP binding site relative to the native substrate. This site contains a pocket lined with hydrophobic residues that cradles the aliphatic tail of IP and controls the size of the isoprene moiety that is bound. ${ }^{8,9}$ Fosfomycin, although smaller than IP, contains a polar nonplanar epoxide group. In FomA, G53, H58, and $\mathrm{S} 142$ create a constellation of donors of hydrogen bonds to the epoxide group and phosphonate groups of fosfomycin. In 
THA IPK and MTH IPK, a glycine replaces $S 149^{31}$ (Figure S1 of the Supporting Information). In addition, the side chains of T79 and K83 enhance the hydrophilicity of the fosfomycin binding site in FomA. The two binding poses observed during dynamics simulations with THA IPK showed that fosfomycin was displaced by a few angstroms relative to IP in the binding site, which might reflect unfavorable interactions between the hydrophobic pocket and the polar epoxide group. In the catalytically productive pose obtained from the dynamics calculations, the epoxide moiety is oriented toward the solvent. In both poses, fosfomycin blocks the binding of IP, consistent with the competitive inhibition profile we observed.

The low level of fosfomycin activity with IPK is consistent with phosphorylation when the molecule is bound in the first pose. In this conformation, a phosphonate oxygen in the antibiotic can form a hydrogen bond with H50, similar to the interaction responsible for orienting IP for nucleophilic attack on ATP. Although the nucleophilic phosphonate group is more distant from the $\gamma$-phosphate group of ATP than the phosphate moiety in IP in the native reaction (Figure $1 \mathrm{~b}$ ), K5, K14, H50, and $\mathrm{K} 205$, which stabilize the transition state for phosphorylation of IP, are engaged in a similar manner in the first pose for fosfomycin. Thus, this pose can be reasonably assigned as the productive ternary complex (Figure 4d). K14 and H50, which are invariant among all IPKs and FomAs, appear to be particularly important. K14 binds the phosphate group of ATP in the IPK.IP.ATP complex and is positioned to stabilize the highly negatively charged transition state. ${ }^{8}$ The K14A mutation results in a doubling of $K_{\mathrm{m}}{ }^{\text {ATP }}$ and a 460-fold decrease in $k_{\text {cat }}$ IP (Figure S8 and Table S1 of the Supporting Information). A K18A mutation of the corresponding residue in FomA inactivates the enzyme. ${ }^{31}$ Both productive and dead-end complexes of fosfomycin with IPK show K14 in almost identical conformations suitable for transition state stabilization (Figure 4d,e). H50 in THA IPK hydrogen bonds with the terminal phosphate groups of IP and the product IPP, ${ }^{8}$ and mutagenesis studies of the corresponding residues in Methanococcus jannaschii IPK (H60) and $S$. wedmorensis FomA (H58) showed that this interaction is important for transition state stabilization accompanying the nucleophilic displacement of ADP., ${ }^{9,31}$ These structural and kinetic data strongly indicate that the first binding pose that contains the H50-phosphonate hydrogen bond is a productive complex.

Our results are consistent with the proposal that FomA might be descended from an archaeal IPK following horizontal transfer of an archaeal ipk gene to Streptomyces and subsequent adaptive mutations that confer antibiotic resistance. Evolution of FomA from IPK by enhancement of the promiscuous fosfomycin kinase activity seen for IPK would provide a selective advantage for the fosfomycin-synthesizing bacterium. ${ }^{4}$ Low levels of activity similar to that we found for fosfomycin kinase activity in IPK $\left(k_{\text {cat }} / K_{\mathrm{m}}=2.6 \mathrm{M}^{-1} \mathrm{~s}^{-1}\right)$ have been shown to confer an immediate selective advantage. For example, phosphonate monoester hydrolase in Burkholderia caryophili $\left(k_{\text {cat }} / K_{\mathrm{m}}=9.5 \mathrm{M}^{-1} \mathrm{~s}^{-1}\right)$ allowed this organism to thrive on glyceryl glyphosate, a previously unencountered phosphorus source. ${ }^{48-50}$ A catalytic efficiency of only $0.3 \mathrm{M}^{-1} \mathrm{~s}^{-1}$ (E. coli glutamine phosphoribosylpyrophosphate amidotransferase) provided a similar advantage, although it was necessary to overexpress the gene from a multicopy plasmid under control of a strong promoter. ${ }^{51}$ Likely instances of horizontal transfer of metabolic genes between Archaea and Bacteria (and other interdomain HGTs) have been proposed for acquisition of mevalonate pathway genes from Archaea (or Eukarya) by Borrelia and Cocci and for acquisition of the bacterial glycerol dehydrogenase gene by Archaea. ${ }^{52}$

\section{ASSOCIATED CONTENT}

\section{Supporting Information}

Detailed experimental procedures for competitive inhibition experiments and site-directed mutagenesis of THA IPK, Michaelis-Menten curves and Lineweaver-Burk graphs, and sequence and structural alignments. This material is available free of charge via the Internet at http://pubs.acs.org.

\section{AUTHOR INFORMATION}

\section{Corresponding Author}

*E-mail: poulter@chem.utah.edu. Telephone: (801) 581-6685.

\section{Funding}

This research was supported by National Institutes of Health Grant GM 25521 (C.D.P.).

\section{ACKNOWLEDGMENTS}

We thank Dr. Kate Slessor for the purified S. wedmorensis FomA used in the product turnover assay. We also thank Dr. James Muller and Dr. Jian-Jung Pan for assistance with the MS/MS fragmentation experiment.

\section{ABBREVIATIONS}

AAK, amino acid kinase; THA, T. acidophilum; IPK, isopentenyl phosphate kinase; FomA, fosfomycin kinase; NAGK, $N$-acetyl-L-glutamate kinase; UMPK, uridine monophosphate kinase; IP, isopentenyl monophosphate; IPP, isopentenyl diphosphate; PDB, Protein Data Bank; MTH, Methanothermobacter thermautotrophicus; PK, pyruvate kinase; $\mathrm{LDH}$, lactate dehydrogenase; Pyr, pyruvate

\section{REFERENCES}

(1) Blume-Jensen, P., and Hunter, T. (2001) Oncogenic kinase signaling. Nature 411, 355-365.

(2) Jensen, R. A. (1976) Enzyme recruitment in evolution of new function. Annu. Rev. Microbiol. 30, 409-425.

(3) Ohno, S. (1970) Evolution by gene duplication, George Allen and Unwin, London.

(4) O’Brien, P. J., and Herschlag, D. (1999) Catalytic promiscuity and the evolution of new enzyme activities. Chem. Biol. 6, R91-R105.

(5) Copley, S. (2003) Enzymes with extra talents: Moonlighting functions and catalytic promiscuity. Curr. Opin. Chem. Biol. 7, 265272.

(6) Yang, K., and Metcalf, W. W. (2004) A new activity for an old enzyme: Escherichia coli bacterial alkaline phosphatase is a phosphitedependent hydrogenase. Proc. Natl. Acad. Sci. U.S.A. 101 (21), 79197924.

(7) Miller, B. G., and Raines, R. T. (2005) Reconstitution of a defunct glycolytic pathway via recruitment of ambiguous sugar kinases. Biochemistry 44, 10776-10783.

(8) Mabanglo, M. F., Schubert, H. L., Chen, M., Hill, C. P., and Poulter, C. D. (2010) X-ray structures of isopentenyl phosphate kinase. ACS Chem. Biol. 5 (5), 517-527.

(9) Dellas, N. P., and Noel, J. P. (2010) Mutation of archaeal isopentenyl phosphate kinase highlights mechanism and guides phosphorylation of additional isoprenoid monophosphates. ACS Chem. Biol. 5 (6), 589-601.

(10) Pakhomova, S., Bartlett, S., Augustus, A., Kuzuyama, T., and Newcomer, M. (2008) Crystal structure of fosfomycin resistance kinase FomA from Streptomyces wedmorensis. J. Biol. Chem. 283, 28518-28526. 
(11) Chen, M., and Poulter, C. D. (2010) Characterization of thermophilic archaeal isopentenyl phosphate kinases. Biochemistry 49, 207-217.

(12) Dela Cruz, F., Garcia-Lobo, J. M., and Davies, J. (2002) in Bacterial resistance to antimicrobials (Lewis, K., Salyers, A. A., Taber, H. W., and Wax, R. G., Eds.) pp 19-36, Marcel Dekker, Inc., New York.

(13) Suarez, J. E., and Mendoza, M. C. (1991) Plasmid-encoded fosfomycin resistance. Antimicrob. Agents Chemother. 35, 791-795.

(14) Bernat, B. A., Laughlin, L. T., and Armstrong, R. N. (1997) Fosfomycin resistance protein (FosA) is a manganese metalloglutathione transferase related to glyoxalase $\mathrm{I}$ and the extradiol dioxygenases. Biochemistry 36, 3050-3055.

(15) Cao, M., Bernat, B. A., Wang, Z., Armstrong, R. N., and Helmann, J. D. (2001) FosB, a cysteine-dependent fosfomycin resistance protein under the control of sigma (W), an extracytoplasmic-function sigma factor in Bacillus subtilis. J. Bacteriol. 183, 23802383.

(16) Etienne, J., Gerbaud, G., Courvalin, P., and Fleurette, J. (1989) Plasmid-mediated reistance to fosfomycin in Staphylococcus epidermidis. FEMS Microbiol. Lett. 52, 133-137.

(17) Pilloff, D., Dabovic, K., Romanovski, M. J., Nonanno, J. B., Doherty, M., Burley, S. K., and Leyh, T. S. (2003) The kinetic mechanism of phosphomevalonate kinase. J. Biol. Chem. 278, 45104515.

(18) Leatherbarrow, R. J. (2001) GraFit, version 5, Erithacus Software Ltd., Horley, U.K.

(19) Zewe, V., and Fromm, H. J. (1965) Kinetic studies of rabbit muscle lactate dehydrogenase. Biochemistry 4 (4), 782-792.

(20) Lindahl, E., Hess, B., and van der Spoel, D. (2001) GROMACS 3.0: A package for molecular simulation and trajectory analysis, pp 306317 , Springer, Berlin.

(21) MacKerell, A. D. Jr., Bashford, D., Bellott, M., Dunbrack, R. L. Jr., Evanseck, J., Field, M. J., Fischer, S., Gao, J., Guo, H., Ha, S., Joseph-McCarthy, D., Kuchnir, L., Kuczera, K., Lau, F. T. K., Mattos, C., Michnick, S., Ngo, T., Nguyen, D. T., Prodhom, B., Reiher, W. E. III, Roux, B., Schlenkrich, M., Smith, J., Stote, R., Straub, J., Watanabe, M., Wiórkiewicz-Kuczera, J., Yin, D., and Karplus, M. (1998) All-atom empirical potential for molecular modeling and dynamics studies of proteins. J. Phys. Chem. B 102, 3586-3616.

(22) Zoete, V., Cuendet, M. A., Grosdidier, A., and Michielin, O. (2011) SwissParam: A Fast Force Field Generation Tool for Small Organic Molecules. J. Comput. Chem. 32, 2359-2368.

(23) Jorgensen, W. L., Chandrasekhar, J., Madura, J. D., Impey, R. W., and Klein, M. L. (1983) Comparison of simple potential functions for simulating liquid water. J. Chem. Phys. 79, 926-935.

(24) Ramon-Maiques, S., Marina, A., Gil-Ortiz, F., Fita, I., and Rubio, V. (2002) Structure of acetylglutamate kinase, a key enzyme for arginine biosynthesis and a prototype for the amino acid kinase family, during catalysis. Structure 10, 329-342.

(25) Davis, I. W., Leaver-Fay, A., Chen, V. B., Block, J., Kapral, G. J., Wang, X., Murray, L., Arendall, B., Snoeyink, J., Richardson, J. S., and Richardson, D. C. (2007) Molprobity: All-atom contacts and structure validation for proteins and nucleic acids. Nucleic Acids Res. 35, W375W383.

(26) Essman, U., Perera, L., Berkowitz, M. L., Darden, T., Lee, H., and Pedersen, L. G. (1995) A smooth particle mesh Ewald potential. J. Chem. Phys. 103, 8577-8592.

(27) Bussi, G., Donadio, D., and Parrinello, M. (2007) Canonical sampling through velocity rescaling. J. Chem. Phys. 126, 012101.

(28) Parrinello, M., and Rahman, A. (1981) Polymorphic transitions in single crystals: A new molecular dynamics method. J. Appl. Phys. 52, $7182-7190$.

(29) Knowles, J. R. (1980) Enzyme-catalyzed phosphoryl transfer reactions. Annu. Rev. Biochem. 49, 877-819.

(30) O'Brian, C. A., and Ward, N. E. (1990) Characterization of calcium- and phospholipid-dependent ATPase reaction catalyzed by rat brain protein kinase C. Biochemistry 29, 4278-4282.

(31) Pakhomova, S., Bartlett, S., Doerner, P. A., and Newcomer, M. (2011) Structural and biochemical insights into the mechanism of fosfomycin phosphorylation by fosfomycin resistance kinase FomA. Biochemistry 50 (32), 6909-6919.

(32) Shoji, J., Kato, T., Hinoo, H., Hattori, T., Hirooka, K., Matsumoto, K., Tanimoto, T., and Kondo, E. (1986) Production of fosfomycin (phosphonomycin) by Pseudomonas syringae. J. Antibiot. 39, 1011-1012.

(33) Lobel, B. (2003) Short-term therapy for uncomplicated urinary tract infection today. Clinical outcome upholds the theories. Int. J. Antimicrob. Agents 22 (Suppl. 2), 85-87.

(34) Cassone, M., Campanile, F., Pantosti, A., Venditti, M., and Stefani, S. (2004) Identification of a variant "Rome clone" of methicillin-resistant Staphylococcus aureus in France: A phylogenetic approach. Microb. Drug Resist. 10, 43-49.

(35) Nakazawa, H., Kikuchi, Y., Honda, T., Isago, T., and Nozaki, M. (2003) Enhancement of antimicrobial effects of various antibiotics against methicillin-resistant Staphylococcus aureus (MRSA) by combination with fosfomycin. J. Infect. Chemother. 9, 304-309.

(36) Kahan, F. M., Kahan, J. S., Cassidy, P. J., and Kropp, H. (1974) The mechanism of action of fosfomycin (phosphonomycin). Ann. N.Y. Acad. Sci. 235, 364-386.

(37) Marquardt, J. L., Brown, E. D., Lane, W. S., Haley, T. M., Ichikawa, Y., Wong, C. H., and Walsh, C. T. (1994) Evidence that the fosfomycin target Cys115 in UDP-N-acetylglucosamine enolpyruvyl transferase (MurA) is essential for product release. Biochemistry 33, 10646-10651.

(38) Kadner, R. J., and Winkler, H. H. (1973) Isolation and characterization of mutations affecting the transport of hexose phosphates in Escherichia coli. J. Bacteriol. 113, 895-900.

(39) Tsuruoka, T., and Yamada, Y. (1975) Characterisation of spontaneous fosfomycin (phosphonomycin)-resistant cells of Escherichia coli B "in vitro". J. Antibiot. 28, 906-911.

(40) Nair, S. K., and van der Donk, W. A. (2010) Structure and mechanism of enzymes involved in biosynthesis and breakdown of the phosphonates fosfomycin, dehydrophos, and phosphinothricin. Arch. Biochem. Biophys. 505 (1), 13-21.

(41) Kobayashi, S., Kuzuyama, T., and Seto, H. (2000) Characterization of the fom $A$ and fom $B$ gene products from Streptomyces wedmorensis, which confer fosfomycin resistance on Escherichia coli. Antimicrob. Agents Chemother. 44, 647-650.

(42) Woodyer, R. D., Shao, Z., Thomas, P. M., Kelleher, N. L., Blodgett, J. A., Metcalf, W. W., van der Donk, W. A., and Zhao, H. (2006) Heterologous production of fosfomycin and identification of the minimal biosynthetic gene cluster. Chem. Biol. 13, 1171-1182.

(43) Marco-Marin, C., Gil-Ortiz, F., and Rubio, V. (2005) The crystal structure of Pyrococcus furiosis UMP kinase provides insight into catalysis and regulation in microbial pyrimidine nucleotide biosynthesis. J. Mol. Biol. 352, 438-454.

(44) Hall, A., and Knowles, J. R. (1976) Directed selective pressure on a $\beta$-lactamase to analyse molecular changes involved in development of enzyme function. Nature 264, 803-804.

(45) Healey, W. J., Labgold, M. R., and Richards, J. H. (1989) Substrate specificities in class A $\beta$-lactamases: Preference for penams vs. cephems. The role of residue 237. Proteins 6, 275-283.

(46) Gibson, R. M., Christensen, H., and Waley, S. G. (1990) Sitedirected mutagenesis of $\beta$-lactamase I. Single and double mutants of Glu-166 and Lys-73. Biochem. J. 272, 613-619.

(47) Imtiaz, U., Manavathu, E. K., Lerner, S. A., and Mobashery, S. (1993) Critical hydrogen bonding by serine 235 for cephalosporinase activity by TEM1 $\beta$-lactamase. Antimicrob. Agents Chemother. 37, $2438-2442$

(48) Van Loo, B., Jonas, S., Babtie, A. C., Benjdia, A., Berteau, O., Hyvönen, M., and Hollfelder, F. (2010) An efficient, multiply promiscuous hydrolase in the alkaline phosphatase family. Proc. Natl. Acad. Sci. U.S.A. 107 (7), 2740-2745.

(49) Ghanem, E., Li, Y., Xu, C., and Raushel, F. M. (2007) Characterization of a phosphodiesterase capable of hydrolyzing EA 2192, the most toxic degradation product of the nerve agent VX. Biochemistry 46, 9032-9040. 
(50) McLoughlin, S. Y., Jackson, C., Liu, J. W., and Ollis, D. L. (2004) Growth of Escherichia coli co-expressing phosphotriesterase and glycerophosphodiester phosphodiesterase, using paraoxon as the sole phosphorous source. Appl. Environ. Microbiol. 70, 404-412.

(51) Patrick, W. M., and Matsumura, I. (2008) A study in molecular contingency: Glutamine phosphoribosylphosphate amidotransferase is a promiscuous and evolvable phosphoribosylanthranilate isomerase. $J$. Mol. Biol. 377, 323-336.

(52) Boucher, Y., Kamekura, M., and Doolittle, W. F. (2004) Origins and evolution of isoprenoid lipid biosynthesis in Archaea. Mol. Microbiol. 52 (2), 515-527.

\section{NOTE ADDED AFTER ASAP PUBLICATION}

This paper was published to the Web on January 11, 2012, with an error in the caption for figure 2. This was corrected in the version published on January 19, 2012. 